\title{
LA DIVERSIDAD SEXUAL Y LA BÚSQUEDA POR LA EFECTIVA IGUALDAD: EN ESPECIAL SOBRE LA HOMOSEXUALIDAD VULNERABILIZADA EN BRASIL
}

\author{
SEXUAL DIVERSITY AND THE SEARCH FOR EFFECTIVE EQUALITY: ESPECIALLY ON \\ LGBTITY VIOLATED IN BRAZIL
}

\section{Douglas Verbicaro Soares}

Universidade Federal de Roraima, Boa Vista, RR, Brasil. E-mail: douglas_verbicaro@yahoo.com.br

DOI: https://doi.org/10.46550/ilustracao.v2i1.61

Recebido em: 06.02.2021

Aceito em: 08.04.2021

\begin{abstract}
Resumen: El estudio tiene como objetivo visibilizar la diversidad sexual humana, en destaque señalar algunos de los obstáculos para las personas homosexuales que viven en situación de vulnerabilidad en la sociedad brasileńa. Así, el presente estudio explicita cuestiones de inclusión de las personas homosexuales y, también, en el combate a la discriminación en el país. Para eso ha sido realizada una investigación basada en fuentes bibliográficas multidisciplinares que retrataron la condena de la homosexualidad en Brasil. La investigación concluye por la existencia de prácticas discriminatorias que dificultan la efectiva integración de las personas LGBTs (lesbianas, gais, bisexuales y transgéneros).
\end{abstract}

Palabras-claves: Condena. Educación. Homosexual. Igualdad.

\begin{abstract}
The study aims to make human sexual diversity visible, noting some of the obstacles for gay people living in vulnerable situations in Brazilian society. Thus, this study sets out issues of inclusion of homosexuals and in the fight against discrimination in the country. For this reason, research has been carried out based on multidisciplinary bibliographic sources that portrayed the condemnation of homosexuality in Brazil. The research concludes with the existence of discriminatory practices that hinder the effective integration of LGBTs (lesbian, gay, bisexual and transgender).
\end{abstract}

Keywords: Sentence. Education. Homosexual. Equality.

\section{Introducción}

Fiable la construcción de un ideal sobre la diversidad sexual, un asunto que abarca Cuna diversidad en sus variantes manifestaciones, relacionadas con el afecto, los deseos y, principalmente, la materialización de conductas, prácticas que involucran la cultura sexual humana, tanto en moldes físicos, como psicológicos. Entender las diversas manifestaciones es fundamental para tornar real el trato igualitario y el respeto de la misma diversidad en el ámbito 
social, sea en Brasil o en cualquier otra parte del mundo.

Empezaremos ese artículo hablando de la diversidad sexual homosexual, uno de los temas más relevantes para nuestra investigación. Para comprender mejor la temática, haremos un relato de la terminología de la propia diversidad, en lo que nos implicaría comentar que su esencia constituye las diversas posibilidades de manifestación de la diversidad sexual humana en Brasil, especialmente relacionada a la homosexualidad.

La orientación sexual homosexual presentada ha sido reivindicada, por manifestaciones sociales, del propio colectivo homosexual, basadas en momentos históricos de la humanidad y en su externalización social evolutiva a lo largo de los tiempos. Por ser un tema multidisciplinar, para hablar de diversidad sexual en Brasil, habrá que estudiar algunas terminaciones como, por ejemplo: la discriminación, el género, la violencia, la sexualidad, la manifestación del deseo homosexual, etc.

La idea de diversidad está intrínsecamente relacionada con la definición de variedad, es decir, la suma de muchas que hacen parte de un conjunto. Para existir la diversidad comentada, son necesarias, por lo menos, dos características diferentes entre ellas. La definición sugerida representa el conjunto de aspectos distintos que están presentes en una materia específica, o sea, en la utilización de las diferencias para componer una aceptación común. ${ }^{1}$ Los derechos sexuales incluyen el derecho de las personas a la integridad de su cuerpo, al pleno desarrollo de su sexualidad, libre de discriminación, violencia o coerción, la búsqueda por el amor y la felicidad.

Para la investigación ha sido empleada una metodología basada en fuente bibliográfica multidisciplinar, con relación con el arte, el derecho, la historia, la psicología etcétera. El estudio tiene cuatro (4) apartados, más la introducción, consideraciones finales y referencias. El primero versa sobre preceptos de la diversidad sexual humana, especial referencia a la homosexualidad. La segunda parte del estudio visibiliza la previsión histórica sobre la sexualidad humana: aspectos de la homosexualidad. La tercera parte de la investigación analiza la búsqueda por la efectiva igualdad entre todos los ciudadanos, explicitando la vulnerabilidad social de las personas LGBTs en Brasil. La última parte de la investigación habla sobre las garantías para los Derechos Humanos y la no discriminación.

De ese modo han sido realizadas algunas preguntas para el estudio: ¿Cómo ha sido tratada la diversidad sexual en el país? ¿La historia habla de la homosexualidad? ¿Las personas homosexuales, así como otros integrantes del colectivo LGBT viven en situación de vulnerabilidad en Brasil? ¿Los Derechos Humanos son respetados en Brasil?

Estas indagaciones han sido las preguntas para el trabajo, que tuvo como principal objetivo el desarrollo de la discusión social sobre la homosexualidad en Brasil.

\section{La Diversidad Sexual Homosexual}

Después de estos comentarios previos, adentraremos en la definición de sexo. ${ }^{2}$ La idea inicial sobre el sexo nos remite a la expresión sexo biológico, que sería el conjunto de informaciones cromosómicas, órganos genitales masculino y femenino. En este caso, el sexo determina que

1 CEZÁRIO, J. Legislação e Jurisprudência LGBTTT. Brasília. Brasil, Instituto de Bioética, Direitos Humanos e Gênero e Associação Lésbica Feminista de Brasília Coturno de Vênus, Brasília, Brasil, 2007, p. 40.

2 ABGLT. Manual de Comunicação LGBT. Lésbicas, Gays, Bissexuais, Travestis e Transexuais. Brasilia, Brasil, 2010, p. 9. 
las féminas tengan un aparato reproductor (vagina) y a los machos (pene), respectivamente. También, según esa vertiente de raciocinio, el sexo corresponde al conjunto de características biológicas y de la anatomía humana que diferencian los hombres de las mujeres. ${ }^{3}$

La terminología caracteriza la capacidad reproductiva y características fisiológicas secundarias que son las responsables por diferenciar el macho de la hembra, de este modo un concepto mucho más biológico, científico que define a todos los animales, no solamente a los humanos. ${ }^{4}$

Sumamos a esa restricta idea que el sexo no puede determinar otras cuestiones que se han dado a conocer, siendo evidenciadas socialmente, con el paso del tiempo, como las referencias de la identidad de género u orientación sexual de las personas. ${ }^{5}$ Para la primera citación, la entenderemos como una variante no dependiente del sexo constituido, es decir, un ser humano puede tener una identidad de género de mujer, de hombre u otras identidades de género posibles, recordando que la identidad de género es una construcción social, y no un signo físico o biológico. ${ }^{6}$ Para la segunda, la orientación sexual de una persona seria la aceptación, la amplia manifestación sexual de una persona de modo que permita su realización plena, afectiva, sentimental.

La orientación sexual homosexual englobaría, según Baile Ayensa: una tendencia interna y estable de desear afectiva y sexualmente las personas de igual sexo. ${ }^{7}$ De acuerdo con esa caracterización, visibilizamos dos ideas importantes, que caracterizan la esencia terminológica de la homosexualidad, son ellas las expresiones: a) Gay: utilizada para englobar los varones homosexuales. Gay es un término anglosajón que originariamente se utilizaba para calificar de "alegres y fiesteros" a ciertas personas. Hoy, en muchos contextos sociales y textos cientificos sirven como sinónimo de persona homosexual. ${ }^{8}$ B) Lesbiana: utilizado para definir las mujeres homosexuales. Lesbiana es la mujer que es atraida afectivamente elo sexualmente por personas del mismo sexol género. ${ }^{9}$

De ese modo entenderemos que la manifestación estable de los sentimientos íntimos de una persona en plasmar sus deseos sexuales hacia a una persona del mismo sexo biológica representaría la esencia de orientación sexual homosexual. En ese caso, si un hombre expresa esa tendencia hacia a otro hombre, se tipificará ese hecho como una manifestación homosexual. Homosexualidad esa utilizada tanto para hombres y mujeres, siendo para los primeros denominados ordinariamente como gais y para las segundas de lesbianas.

Con base en estos argumentos, la sexualidad humana homosexual será vista como una más que puede tener la amplitud que envuelve en tema y, no sólo la que se manifiesta en la mayoritaria, es decir, el modelo heterosexual dominante. ${ }^{10}$ Otra manifestación presente es la bisexual, que se caracteriza en el momento en que una persona sienta atracción sexual o afectiva por otra persona de su mismo sexo y, también, por el sexo opuesto. Bisexual es la persona que se

3 CANCIO ÁlVAREZ, M. D. "Glosario". In: Conde Álvarez, E. / Figueruelo Burrieza, A. / Nuño Gómez, l.: Estudios Interdisciplinares sobre Igualdad. Segunda Edición. Madrid, España: Iustel - Instituto de Derecho Público, 2011, p. 306.

Cf. CANCIO ÁLVAREZ, M. D. "Glosario", loc. cit., p. 305.

CEZÁRIO, J. Legislação e..., op. cit., p. 35.

CEZÁRIO, J. Legislação e Jurisprudência LGBTTT..., op. cit., p. 38.

BAILE AYENSA, J. I. Estudiando la Homosexualidad. Teoría e investigación. 1a Edición, Madrid, España: Ediciones Pirámide, 2008, p. 34.

8 BAILE AYENSA, J. I. Estudiando..., op. cit., pp. 30-1.

9 ABGLT. Manual de Comunicação LGBT..., op. cit., p. 14

10 ABGLT. Manual de Comunicação LGBT..., op. cit., p. 9. 
relaciona afectiva y sexualmente con persona de ambos los sexos/géneros. Bi es una forma reducida de hablar de las personas con la orientación del deseo bisexual. ${ }^{11}$

En ese sentido, las personas que desarrollan eses comportamientos y expresan sus emociones, tanto a personas del sexo diverso como a las del mismo sexo biológico. También se puede argüir que esa diversa tipificación de la sexualidad humana acaba siendo cuestionada, muchas veces, pues socialmente se tiende a dudar que las personas puedan direccional sus afectividades hacia a los dos sexos y que sería un modo de encubrir sus verdaderos sentimientos, es decir, que acabarían siendo bisexuales pues, socialmente, no les permitiría moralmente que se expresasen como realmente son, personas homosexuales.

No estamos de acuerdo con esa visión, creemos sí que una persona pueda desarrollarse emocionalmente y decidir amar o a practicar conductas sexuales tanto con hombres, como con mujeres, teniendo en esas conductas la satisfacción personas que buscan para ser felices. Si en un determinado momento se sienten mejor al lado de una persona del mismo sexo, eso no impide que en un futuro no se pueda encontrar ese placer junto a una persona del sexo distinto. Lo importante aquí es la preservación de una esencia primordial, el placer humano, que no debe estar bajo la influencia de jerarquías o roles que imponen los deseos sexuales humanos a ser seguidos.

\section{La previsión histórica sobre la sexualidad humana: aspectos de la homosexualidad}

Para Preciado, al hacer referencia a la cuestión de la diversidad sexual, a lo largo de la evolución social de la humanidad, nos comenta que el asunto ha tenido en los últimos siglos, una relevante aportación didáctica. Según sus ideas, en las cuales estamos de acuerdo: es necesario pensar el sexo, al menos a partir del siglo XVIII, como una tecnología biopolítica. Es decir, como un sistema complejo de estructuras reguladoras que controlan la relación entre los cuerpos, los instrumentos, las máquinas, los usos y los usuarios. ${ }^{12}$ De ese modo, visibilizamos la cuestión del sexo incorporado a la evolución de la humanidad, en muchas sociedades, es decir: la sexualidad pasa a ser entendida como un tema esencial para la vida de las personas, no solamente relacionada a la reproducción humana, pero al reconocimiento social que el sexo sería algo mucho más amplio, relacionado desde el placer individual de cada persona, hacia la satisfacción física y mental de cada individuo y su vínculo en el entorno a lo que vive.

Con esa línea de interpretación ampliamos la tipología de la diversidad sexual a no sólo a las propias características específicas y biológicas del aparato reproductor femenino y el masculino, su funcionamiento y los caracteres sexuales secundarios que derivan de las hormonas. ${ }^{13}$ Este hecho nos posibilitará abordar la temática homosexual como un concepto complementario a esa diversidad existente.

Recordamos que la amplitud del tema sobre la sexualidad humana está relacionada con:

Elaboraciones culturales sobre el placer y los intercambios sociales y corporales que comprenden desde el erotismo, el deseo, hasta nociones relativas a la salud, la reproducción, al uso de tecnologías y el ejercicio del poder en sociedad. Las definiciones actuales de la sexualidad abarcan, en las ciencias sociales, significados,

11 ABGLT. Manual de Comunicação LGBT..., op. cit., p. 11.

12 PRECIADO, B. Manifiesto Contrasexual. Colección Argumentos. Editorial Anagrama. Barcelona, España, 2011, p. 68.

13 PRECIADO, B. Manifiesto..., op. cit., p. 68. 
ideas, deseos, sensaciones, emociones, experiencias, conductas, prohibiciones, modelos y fantasías que son configurados de modos diversos en distintos contextos sociales y periodos históricos. Es un concepto dinámico que va evolucionando y que está sujeto a diversos usos, múltiples y contradictorias interpretaciones y que se encuentra sujeto a debates y a disputas políticas. ${ }^{14}$

De esta manera, notaremos que, a lo largo de la historia, la comprensión sobre la sexualidad humana amplía sus horizontes, haciendo con que la diversidad existente sobre el lema tome rumbo hacia a nuevas descubiertas, añadiendo distintos preceptos para justificar la generalidad de ese tema, que todavía necesita ser estudiado más a fondo, con el objetivo de conocerlo justo por su magnitud e importancia que tiene para la propia humanidad.

Es importante aclarar que la Declaración Internacional, que versa sobre el reconocimiento y la protección de los Derechos Humanos en los temas de los derechos a la sexualidad. Declaración esa, fruto del resultado del Congreso Mundial de Sexología realizado en Espańa, más específicamente en Valencia, en el año de 1997. Posteriormente, ha sido revisada y aprobada por la Asamblea General de la Asociación Mundial de Sexología (WAS - World Association for Sexology) en 1999, en el $14^{\circ}$ Congreso Mundial de Sexología realizado en la ciudad china de Hong Kong. ${ }^{15}$ Declaración Internacional fundamental que implica en el reconocimiento en el ámbito globalizado de la importancia que asume los temas de la sexualidad humana y, también, en los mecanismos para la protección y efectividad de los derechos relacionados con la temática.

Relacionado a los argumentos aportados hasta ese momento, citaremos a Furlani: por más que esta Declaración ha sido elaborada en el sentido generalizado, en que dice respeto a las diversas identidades sexuales, la Declaración mencionada representa un documento político, de reivindicaciones, conquistas, reconocimiento a los grupos e/o sujetos subordinados y de respecto a ellos. Para la autora:

Los derechos sexuales son derechos humanos universales basados en la libertad, dignidad e igualdad de todos los seres humanos. Una vez que la salud sexual es un derecho fundamental, la salud sexual debe de ser un derecho humano básico. Para asegurarnos que los seres humanos y la sociedad desarrollen una sexualidad saludable, los derechos sexuales, a seguir, deben de ser reconocidos, promovidos, respectados y defendidos por todas las sociedades de todas las maneras. Salud sexual es el resultado de un ambiente que reconoce, respecta y ejercita estos derechos sexuales. ${ }^{16}$

Preciado también hace referencia a la temática de la sexualidad como el fruto de la construcción del comportamiento humano sociopolítico, influenciado por el tiempo histórico. Es un concepto amplio, de diferentes manifestaciones que no solamente está en la división estricta del masculino y femenino. Es algo que está más allá de estos dos términos, existe sí la necesidad de considerar una serie de factores que influyen en la manifestación sexual del cuerpo humano, sea por motivos biológicos (genéticos o cromosómicos) o por otras variantes. ${ }^{17}$

Construcción esta que versa respecto a los hábitos, prácticas, comportamientos,

14 ABGLT. Manual de Comunicação LGBT..., op. cit., p. 9.

15 VERBICARO SOARES, D. Homossexualidade e Forças Armadas. A busca pela efetividade dos Direitos Humanos no Brasil. Trabajo de Grado. Universidad de Salamanca: Programa de Doctorado Pasado y presente de los Derechos Humanos, Salamanca, España, 2011, p. 52-3.

16 VERBICARO SOARES, D. Homossexulidade..., op. cit., p. 54.

17 FURLANI, J. "Direitos Humanos, Direitos Sexuais e pedagogia queer: o que essas abordagens têm a dizer à educação sexual?”. In: Diniz Junqueira, R. Diversidade Sexual na Educação: problematizaçóes sobre a homofobia nas escolas. Coleção Educação para todos. Brasília, Brasil, 2009, p. 302. 
pensamientos de una persona con la temática del sexo y su posterior relación con el medio social. Varias son las áreas que procuran definir y estudiar la sexualidad humana, desde la biología, pasando por la teología, psicoanálisis, sociología, antropología, derecho ${ }^{18}$ y muchas otras.

Complementando las ideas de Preciado, al definir las características de la sexualidad humana se extiende de esta manera: el ser humano inicia su historia cuando el proceso de desarrollo de las diferentes especies sexuadas llevaba un largo camino, ${ }^{19}$ compartiendo con todas las especies sexuadas algunas características esenciales, entre ellas: las que destacan la identidad sexual (fémina y macho), roles sexuales en la reproducción y creencia, afectos sexuales, como el deseo y la atracción, y los afectos sociales, como los vínculos entre progenitores y crías. Pero, este proceso adquiere, en el caso de nuestra especie, características propias, que suponen una diferenciación cualitativa del resto de las otras especies. ${ }^{20}$

Los seres humanos, en efecto son, 1) individuos diferentes genética y culturalmente, 2) tienen una identidad sexual que depende de las características biológicas del hombre y de la mujer, 3) tenemos una identidad sexual sobre las que las otras culturas han construido unos roles de género, casi siempre discriminatorios para con las mujeres, que regulan numerosos aspectos de la vida social de las personas, 4) tenemos, como ocurre en otras especies sexuadas, una fuerte motivación sexual (deseo y atracción) y su actividad sexual está premiada (placer sexual), 5) tenemos la característica de que la sexualidad es una dimensión más rica y compleja que nos limita a una mera función reproductora. ${ }^{21}$

No se puede encerrar ese parte del trabajo sin hacer alusión al movimiento feminista ${ }^{22}$ moderno, que como ya ha sido ejemplificado con Preciado, también denominado nuevo movimiento, o la $3^{a}$ ola del feminismo ${ }^{23}$, tiene que ver con el período que comienza, según la opinión de Cristina Garaizabal, en los años sesenta.

Para complementar esa idea, es posible citar, por ejemplo, la situación de España frente a cambios en el modelo del activismo feminista en el país ibérico, principalmente a partir del ańo 1975. Momento ese marcado en la historia con la muerte del General Francisco Franco, personaje singular del Gobierno, que durante décadas implementó, como diversos en Europa o en América, el fortalecimiento de un modelo militar de represión a las minorías, entre ellas los homosexuales que han sido perseguidos por el Régimen de Franco.

Después de este enfoque sobre la historia en el país europeo mencionado, el movimiento feminista novedoso representó, por cierto, un cambio fundamental en la comprensión del género como construcción social. En este sentido, hemos encontrado en las palabras de Preciado algunas de las respuestas sobre esa nueva etapa de las reivindicaciones feministas de igualdad de trato en relación con los hombres y la libertad sexual. Principalmente en los modelos impuestos socialmente, o sea, basados en un padrón heterosexual dominante, que reducía la liberación de las mujeres en los ámbitos públicos y privados. Si hablamos de las mujeres también tenemos que

18 Cf. FURLANI, J. "Direitos Humanos..., loc. cit., p. 302.

19 VERBICARO SOARES, D. Las mujeres y las personas homosexuales en las Fuerzas Armadas. Especial referencia a las FFAA brasileñas. Trabajo fin de curso Máster Oficial en Estudios Interdisciplinares de Género. Universidad de Salamanca. Salamanca, España, 2012, p. 62.

20 VERBICARO SOARES, D. Las mujeres..., op. cit., p. 62.

21 LÓPEZ SÁNCHEZ, F. La Educación Sexual. Madrid, España: Biblioteca Nueva, 2005, p. 20-22.

22 Cf. CANCIO ÁLVAREZ, M. D. "Glosario", loc. cit., p. 305.

23 YOUTUBE. Beatriz Preciado. 16 de julio de 2006. Disponible en: <http://www.youtube.com/watch?v=W8wfc2JNMd4>. Acceso en: 26 de may de 2021. 
tratar sobre las mujeres-lesbianas, que acababan minimizadas doblemente.

Es válido recordar que, durante la $2^{\text {a }}$ Guerra Mundial, hubo alteraciones en los modelos o roles de las mujeres, pues empezaran a ser utilizadas como mano de obra en la esfera del público, ya no solamente como amas de casa y de cuidadoras familiares. Las mujeres adentran en los espacios que antes eran permitidos solamente a los hombres. Con eses hechos la visualización de la participación femenina abre camino a la lucha por reivindicaciones por mejores situaciones de vida para las mujeres.

Para Probst sobre la situación de las mujeres en la historia:

Las mujeres, a lo largo de la historia, sufrieron y todavía sufren discriminación por los más diversos motivos (religiosos, biológicos, políticos, económicos, culturales, por orientación sexual, etcétera), recibiendo salarios más bajos, sufren con los techos de cristal que les impiden ascender a los cargos más elevados en una determinada profesión, asedios y acosos en el ámbito laboral. La lucha de las mujeres contra los preconceptos y las discriminaciones continúa y sigue siendo actual, con el objetivo de conquistar y demostrar su fuerza, su capacidad profesional, comprobando que el hecho de pertenecer a un sexo específico no les invalida o las coloca en situación de menos valía al sexo masculino. ${ }^{24}$

La lucha por evitar esas distintas formas de discriminación ha estado (en los últimos siglos) en las bases de las reivindicaciones sociales históricas más destacables, desde la Revolución Francesa, pasando por la Revolución Industrial, hasta los clamores más actuales para poner fin a los obstáculos que dificultan el trato igualitario entre los ciudadanos.

Para esa realidad, las reivindicaciones feministas de cambios, principalmente a partir de los años $70 \mathrm{y}$, también posteriores, han repercutido en romper con los ideales pos-femininos, retratando las discusiones sobre la igualdad, la diferencia, la justicia. De ese modo, ampliando las definiciones de género y sexo, sus características, sus identidades e instrumentos que permitan descubrir la sexualidad humana en sus diferentes manifestaciones en diferentes sociedades, principalmente en Brasil. ${ }^{25}$

Esa alteración será marcada por la manifestación del modelo feminista más liberal, de luchar por lo logro de la igualdad entre los sexos, identificando uno de los principales problemas enfrentados: la exclusión de las mujeres de la vida pública e inserción en el mercado de trabajo , visión presente en las ideas de Simone de Beauvoir así como en las nuevas exigencias de un futuro con la revolución tecnológica, las relaciones entre las personas, las máquinas y la tecnología, como en los argumentos de Donna Haraway. ${ }^{26}$ Ya para McDowell:

La investigación feminista tiene una importante tarea que cumplir: mostrar la variación de esas relaciones de género y poder, su tendencia a la reproducción y las posibles formas de impedirlo. En ese sentido, podemos comprometernos con el proyecto de superar las limitadas formas de ser hombre o mujer que pretendemos imponernos. ${ }^{27}$

24 PROBST, E. R. A evolução da mulher no mercado de trabalho. Instituto Catarinense de Pós-Graduaçáo - ICPG. Curso de Gestão Estratégica de Recursos Humanos. Florianópolis/SC, Brasil, 2003, p. 1.

25 VERBICARO SOARES, D. Las mujeres..., op. cit., p. 69.

26 Preciado con su obra se ha dado a conocer y tornándose relevante para el movimiento feminista actual, relacionados con la sexualidad humana y sus manifestaciones en sociedad, principalmente cercanas a los temas queer. En su momento mencionamos que para Preciado: la teoría queer va a aparecer como una vuelta reflexiva sobre los errores del feminismo, resultando en una nueva visión del movimiento feminista, adaptando las ideas de variados autores llegando hasta Haraway, en su manifiesto cyborg. VERBICARO SOARES, D. Las mujeres..., op. cit., p. 69.

27 MCDOWELL, L. Género, identidad y lugar. Madrid, España: Ediciones Cátedra, 1999, p. 363. 
La frase de Simone de Beauvoir "no se nace mujer, se llega a serlo" inaugura una nueva época del feminismo moderno, una de cuyas preocupaciones fundamentales va a ser la crítica a la supuesta naturalidad de los géneros, así como analizar y desvelar los múltiples mecanismos a través de los cuales se construyen éstos.

En el mismo sentido que al mencionar la evolución del movimiento feminista más reciente, posiciona el ideario de Preciado como un momento de descubrimiento de la sexualidad humana en confronto con los roles previamente establecidos en sociedad. Donde el heterosexismo predomina e impone su fuerza sobre las diversas manifestaciones sexuales humanas, como el más importante y supuestamente natural, basado en ideas patriarcales, machistas, de perjuicios, discriminación y etcétera. Hay que recordar que Preciado ha visibilizado mundialmente la problemática queer, relacionada con la conquista de derechos de la comunidad LGBT e igualdad de trato para ese colectivo social. ${ }^{28}$

El cuerpo queer pone en cuestión la mitología que garantiza la naturalización de la filiación y la diferencia sexual. En este contexto, el sujeto queer genera todo un conjunto de angustias sexuales y políticas. La cuestión de la recepción de las teorías queer en Francia o en España no me preocupa en absoluto porque los límites nacionales no me parecen, en efecto, pertinentes para pensar el trabajo queer. ${ }^{29} 30$

Con base en estas ideas, Preciado define su trabajo como una traducción política de determinados trabajos científicos: una de las cuestiones politicas más urgentes hoy es precisamente la que suscita la relación entre las politicas de identidad y las estrategias queer. Por más que tenga recibido las más variadas críticas, positivas y negativas cuanto, a su iniciativa literaria, es muy importante aducir que, en los últimos tiempos, se ha incrementado la visibilidad de muchas personas que antes eran aisladas de participación social. ${ }^{31}$ Entre esa visibilidad están las lesbianas y el reconocimiento social de que cada persona es libre para tener control sobre sus cuerpos, su sexualidad, los placeres que eso implica y los modos de buscar la libertad que cada persona en su esencia, su dignidad de querer asumirse como cada uno es, sin tener violada esa esencia.

Por esa razón ha sido indispensable la influencia del movimiento feminista y la apertura de la participación femenina reivindicativa por una verdadera igualdad de condiciones, de trato hacia a los hombres, del mismo modo que se planteaban una mejor inclusión de las mujeres en el ámbito laboral diversos del hogar.

Entendemos que son relevantes los instrumentos que visibilicen los considerados, por muchos, como "asuntos olvidados o considerados como menos relevantes". No estamos para nada de acuerdo con la limitada visibilidad extendida a las discusiones sobre género y sexualidad humana, una vez que son temas actuales, de importancia nacional e internacional y que necesitan ser protegidos y hechos efectivos en la construcción de políticas de integración emancipadora de los colectivos victimados que viven en exclusión social.

La historia evidenció que después de la revolución industrial, la esfera laboral de las mujeres, pasando de una vida de cuidadora de la familia, predominantemente, en las tareas de la casa y de la familia a una inclusión de las mujeres en las mujeres en un régimen capitalista de

28 VERBICARO SOARES, D. Las mujeres..., op. cit., pp. 69-70.

29 VERBICARO SOARES, D. Las mujeres..., op. cit., p. 70.

30 PRECIADO, B. Manifiesto Contrasexual. Colección Argumentos. Barcelona, España: Editorial Anagrama, 2011, p. 83.

31 SCRIBD. Entrevista con Beatriz Preciado por Jesús Carrillo. Disponible en: <http://es.scribd.com/doc/62741654/Entrevistacom-Beatriz-Preciado-por-Jesus-Carrillo>. Acceso en: 26 de may de 2021. 
utilización del trabajo de las mujeres como fuente generadora de beneficios para los empleadores. En este sentido, se ha visto la concentración doble actividades para ellas, que han tenido que asumir las funciones tipificadas como femeninas del ámbito privado, doméstico y de la familia, añadiéndole la participación en la esfera pública, trabajando para ayudar a complementar las necesidades básicas de la vida.

También encontraremos en las bases del movimiento feminista (1848), con la creación de la Convención de los Derechos de la Mujer de Nueva York. Por otro lado, se puede argumentar que las semillas de las luchas reivindicativas a favor de una igualdad de trato entre hombres y mujeres se acentuó con la implementación de la Revolución Francesa, que bajo la protección de los principios de la fraternidad, igualdad y libertad han cambiado el panorama social y que, hasta la actualidad, sus ideas siguen presentes en las reivindicaciones de los grupos en situación de vulnerabilidad social participativa por el mundo.

A lo largo de los últimos siglos, el movimiento feminista se hace más sólido y junto con otros, como el de los trabajadores, la reivindicación por la igualdad de condiciones y derechos entre los sexos se materializa de modo más efectivo. Así, las mujeres hacen escuchar sus clamores por un trato igualitario, cuestionado sobre todo el supuesto rol discriminatorio del sexo como justificante absoluto de las tareas de trabajo y los derechos atribuidos a los hombres y a las mujeres.

Teniendo en cuenta que el sexo biológico ya no podía seguir siendo usado como motivo de exclusión social participativa de las mujeres. El proceso de toma de conciencia femenina ha motivado su lucha para posibilitar los cambios necesarios para el modelo social constituido, predominantemente paternalista.

Brasil acompañó las reivindicaciones por el mundo, siendo el movimiento feminista de este país inspirados en lo que estaba ocurriendo en Europa y Norte América. Como no sería distinto de otros países del continente suramericano, bajo una grande influencia de instituciones conservadoras, como la iglesia, por ejemplo, las mujeres habían tenido que acostumbrarse a un supuesto modelo de sumisión al hombre, sin razón alguna para los defensores de esa idea restrictiva sobre género.

Como ya mencionado anteriormente, con la idea de que las mujeres eran educadas para el cuidado: de la casa, de la familia, de casar y reproducir. De este modo, perpetuando las bases de una ideología cristiana erróneamente interpretada en desfavor de la igualdad. En la experiencia brasileña, reforzando los roles estereotipados de madre y mujer débil, que necesitaba la protección de una figura masculina para su propia subsistencia, sea inicialmente por un padre y luego a un marido.

Para cambiar ese modelo, el movimiento feminista, buscando la emancipación de las mujeres en el ámbito público y privado, reivindicaba la igualdad de trato en relación con los hombres. Primero en poder votar, de estudiar, de decidir con quien casarse, de separarse, de trabajar, de tener un salario digno y proporcional a cualquier actividad desarrollada por un hombre. Es decir, la conquista por una real participación ciudadana en igualdad de condiciones con los hombres.

En Brasil es válido mencionar el papel que tuvo Berta Lutz, que, en la década de 20, del siglo pasado, creó la Federación Brasileña por el Progreso Femenino, que reforzaban las 
ideas previamente mencionadas. Con el paso de la historia, las reivindicaciones se extienden a la sexualidad humana, como, por ejemplo, tener derecho a tener hijos y de planear la constitución familiar. No podemos dejar de considerar que un avance importante ha sido la conquista por el placer sexual de la mujer y de una mayor libertad sobre su propio cuerpo. ${ }^{32}$

Basándose en esa realidad, el movimiento feminista empieza a tratar de temas relacionados con la orientación u identidad sexuales. La visualización de las lesbianas en sociedad se plasmará en las décadas posteriores. En este momento hacemos una crítica al feminismo que por luchar por la igualdad de trato de las mujeres en relación con los hombres. Actualmente, las mujeres lesbianas se han deparado con una cierta dificultad en una lucha común junto con las mujeres heterosexuales.

En su momento, el movimiento homosexual ha estado unido a las marchas femeninas en la conquista de la igualdad, pero que después, las lesbianas han tenido más difícil la garantía de sus derechos, principalmente en las décadas de los años 70 y 80 debido a la característica extendida a los homosexuales como agentes del pecado, enfermos y transmisores del virus del SIDA. ${ }^{33}$

En este sentido, complementando la participación del movimiento feminista a partir de los 80, así como la lucha del colectivo de las lesbianas, han buscado unir los objetivos en cambiar realidades. Por esta razón, en la década que se presentó después, se trabajó las cuestiones de género y de las ideas sobre la naturalidad de las cosas o de una mayoría, como el modelo heterosexual predominante.

Según Herrero Brasas, los avances sobre los temas relacionados con la cultura denominada queer, que tiene que ver con la temática de los homosexuales en España ${ }^{34}$, donde la participación ciudadana organizada, ha podido hacer, de este país, todo un referente en la temática del reconocimiento de derechos a las personas con esa orientación sexual. Equivalentemente ha permitido la consecución por un trato igualitario en la sociedad española, como un ejemplo de éxito. La experiencia española como un modelo de integración para la realidad de exclusión brasileña.

\section{La búsqueda por la efectiva igualdad}

Con la efectividad de un modelo jurídico de protección a ese colectivo social minoritario, la lucha por el reconocimiento de derechos puede ser visibilizado por medio de la obra "Primera Plana” un camino novedoso para el colectivo LGBT:

En España el movimiento de liberación gay y lesbiano ha conseguido una meta casi única en el mundo. Es algo sobre lo que merece la pena reflexionar desde diferentes perspectivas. Las colaboraciones de este libro ofrecen la perspectiva humana, subjetiva, íntima, de los activistas, de aquellos que han liderado la lucha por la igualdad desde los distintos frentes. ${ }^{35}$

32 VERBICARO SOARES, D. Las mujeres..., op. cit., p. 69.

33 VERBICARO SOARES, D. La libertad sexual en la sociedad: especial referencia a la homosexualidad en las Fuerzas Armadas Brasileñas. Salamanca, España. Tesis Doctoral. Universidad de Salamanca - USAL: Programa de Doctorado Pasado y Presente de los Derechos Humanos, 2015, p. 186.

34 VERBICARO SOARES, D. La libertad, op. cit., p. 186.

35 XEGA.ORG. Juan Antonio Herrero Brasas publica su nuevo libro 'Primera Plana. La construcción de una cultura queer en España.' Disponible en: <http://xega.org/xega/noticias/xega/juan_antonio_herrero_brasas_publica_su_nuevo_libro_ primera_plana_la_construccion_de_un>. Acceso en: 26 de may de 2021. 
La propia comunidad LGBT al hablar de temas sobre la identidad de género y, también, a través de los estudios de género tienen una proyección discursiva en la actualidad y, así, hacen con que la obra de Preciado se coloque dentro de este giro de ideas, haciendo con que nuevas discusiones salgan adelante en los más variados sectores: políticos, económicos, sociales, jurídicos, educativos, etcétera. Por así decir, ocurre un giro con relación al régimen de ocultamiento y secreto que se basaban las ideas disciplinares antiguas del siglo XIX, que se produjeron con las identidades sexuales (homosexual y heterosexual).

Verdaderos cambios vienen siendo notados, pasando desde la criminalización social de las identidades de géneros hasta la conquista y el reconocimiento del trato igualitario entre todos. Para Preciado, un momento de progresiva normalización y asimilación de las minorías sexuales en la norma (matrimonio gay, igualdad legal, representación mediática) parece politicamente incongruente seguir considerando a los gais y las lesbianas como un grupo politico radical. ${ }^{36}$

En años anteriores citamos a Martínez Quinteiro: el derecho es un producto histórico y necesita de análisis histórico para ser comprendido. ${ }^{37}$ Es válido decir que el movimiento feminista, originario en Europa por pensadoras como Simone de Beauvoir (años 60) y Betty Friedan en los EE.UU., han contribuido para que millones de mujeres pudiesen auto representar y tener una real participación para luchar por cambios que colocasen fin a una situación de desigualdad en la que se encontraban en relación a los hombres. ${ }^{38}$

Para las situaciones de vulnerabilidad y a los obstáculos enfrentados tanto por las mujeres, así como para los homosexuales (gais y lesbianas), bisexuales, transexuales y travestís son sujetos de la historia y, por eso, forman parte indispensable para la consolidación de una sociedad más justa e igualitaria. Haremos referencia a las palabras de Paolo Zanotti sobre la homosexualidad y los obstáculos para vivir esa orientación sexual. Según el autor:

Los homosexuales han vivido durante siglos en un mundo misteriosos y reticente, un mundo un poco teatral hecho de travestimos, matrimonios de tapadera y puntos de encuentro nocturnos poco recomendables. Un mundo de palabras misteriosas. Conocidas sólo por la comunidad homosexual, con un doble significado (uno para los iniciados, otro para la sociedad normal); un mundo de palabras - algunas brutales. Otras eufemísticas - usadas por los "normales" para referirse a los "diferentes". ${ }^{39}$

La obligación de respetar los derechos y los deberes, de tener ciudadanía constituye un importante papel que pertenece a cada individuo en sociedad. Es válido resaltar que el papel desempeñado por los hombres y las mujeres en sociedad se ha manifestado de manera diversas para ellos. De ese modo, la problemática estaría en la importancia que les atribuye mayor o menor grado de valor en las acciones desempeñadas por las mujeres y, igualmente, aplicable a las personas victimadas por tener una orientación del deseo sexual distinta de la mayoría heterosexual. ${ }^{40}$

La búsqueda por la igualdad de trato entre hombres y mujeres, las reivindicaciones feministas, la lucha por instrumentos legales de protección de derechos, por motivos

\footnotetext{
SCRIBD. Entrevista con Beatriz Preciado por Jesús Carrillo, op. cit., Consultado el 16 de Noviembre de 2014.

VERBICARO SOARES, D. Las mujeres..., op. cit., p. 70.

VERBICARO SOARES, D.: Las mujeres..., op. cit., pp. 70-1.

39 ZANOTTI, P. Gay. La identidad homosexual de Platón a Marlene Dietrich. Colección Noema. Madrid, España: Turner Publicaciones, 2007, p. 9.

40 VERBICARO SOARES, D. Las mujeres..., op. cit., p. 71.
} 
fundamentales (cuando las normas estén previstas y reconocidas legalmente en un Estado), o cuando están reconocidos como universales en disposiciones internacionales. Todas ellas tienen sus orígenes en solicitudes sociales y basadas en hábitos, costumbres que cambian en el tiempo. Es importante que estos derechos estén pasando por estructuraciones conforme la real necesidad de una sociedad, caso contrario, serían solamente normas sin real efectividad. ${ }^{41}$

Es válido recordar que la violencia contra este grupo social, así como ocurre con las minorías (sea por orientación sexual, por creencia religiosa, cultural, etcétera) ha estado presente en las sociedades, pero su comprensión parece estar olvidado. Hecho este, con el pasar de la historia, basado en diferentes reivindicaciones (económicas, sociales, políticas, culturales) abren camino para un cambio en el comportamiento. Los temas que antes no eran hablados socialmente, como han sido los temas de la sexualidad humana y, también, sobre la igualdad de trato entre hombres y mujeres abren camino al respeto, al reconocimiento de la diferencia y a la búsqueda por la protección y el reconocimiento efectivo de esta igualdad. ${ }^{42}$

Para notar verdaderos cambios en las realidades problemáticas relatadas en ese estudio es indispensable la sensibilización sobre la igualdad de trato entre los géneros. Todos los esfuerzos en conseguir la concientización general de estas sociedades, donde los hombres, detentores de una forma de dominación más amplia, puedan permitir que los homosexuales (hombres y mujeres) tengan su efectiva participación en los más diversos sectores. Por esta razón la visibilidad de este colectivo socialmente es importante para romper tabús. ${ }^{43}$

Para corroborar con esa idea Valladares explicita que: en la actualidad, las mujeres están pensando en una reconceptualización de la ciudadanía, no a partir de la noción tradicional, pues desde allí siempre seremos ciudadanas de segunda clase. El pensamiento de la ciudadanía de las mujeres está ligado a lucha por ser sujetos de su propia historia, lo que implica tener y ejercer derechos, pues la ciudadanía plena, se expresa justamente en las posibilidades de participación, exigibilidad y ejercicios de derechos. ${ }^{44}$

Los derechos sexuales tienen una dupla dimensión, tanto afectan la esfera íntima de la persona, como también en los niveles colectivos por lo que inciden en políticas públicas, nacionales, regionales y mundiales. Pensar en el tema de la ciudadanía objetiva superar la visión restringida que rodea la temática de la ciudadanía en el ámbito político: los particulares en relación con el poder del Estado, pues sus elementos básicos son: participación, representación y poder, tanto en el público, como en el privado. De ahí proviene la idea de que la reivindicación por derechos sexuales representa un proceso de ampliación de la ciudadanía, así como de un reconocimiento de la diversidad. ${ }^{45}$

En este sentido, son necesarios cambios en las más diversas áreas: sociales, políticas, jurídicas, económica, etc. Cambios que están dentro del propio ideal del movimiento feminista, que representa su esencia en el reconocimiento de la no superioridad de la figura de la mujer en relación con el hombre, como muchos defienden, pero verdaderamente un igual trato entre los

41 VERBICARO SOARES, D. Las mujeres..., op. cit., pp. 71-2.

42 VERBICARO SOARES, D. Las mujeres..., op. cit., pp. 71-2.

43 VERBICARO SOARES, D. La viabilidad de la presentación de un servicio contratado para un establecimiento de alojamiento turístico en Salamanca para alojar estudiantes brasileños y gestión de sus asuntos académicos. C.F.G.S. Gestión de Alojamientos Turísticos. Escuela de Hostelería "Fonda Veracruz". Modulo Proyecto Final. IES Vaguada de la Palma. Salamanca, España, 2014, p. 45.

44 VERBICARO SOARES, D. Las mujeres..., op. cit., p. 72.

45 VERBICARO SOARES, D. Las mujeres..., op. cit., p. 72. 
sexos. ${ }^{46}$

Por esta razón, los ensayos científicos ${ }^{47}$ sobre las relaciones de género y de sus identidades ${ }^{48}$ contribuyen para comprender las dificultades que sufren las personas homosexuales en sociedad y, también, el difícil trabajo de los movimientos con ideales de igualdad entre los géneros. Como en los esfuerzos de estudiar sus problemáticas y buscar alternativas para retirar los obstáculos que general situaciones de vulnerabilidad a esas personas.

Estos cambios han sido posibles pues la "anormalidad" femenina ha sido entendida como una construcción de la cultura patriarcal subordinante para las mujeres que puede y debe ser reconstruida con una propuesta de igualdad de trato entre los géneros. ${ }^{49}$

Aprovechamos ese raciocinio y complementamos que la reivindicación de otros colectivos en semejante situación de vulnerabilidad, como son los homosexuales, han estado, en las últimas décadas, luchando junto con el movimiento feminista para lograr alcanzar una real igualdad de trato y participación ciudadana, reformulación y cambio del conjunto político del Estado del bienestar brasileño. ${ }^{50}$

Del mismo modo De Sousa Santos, al analizar el tema de las reivindicaciones feministas que, en los últimos veinte años, en las concepciones dominantes de las diferentes ciencias, apuntó que la naturaleza es un mundo de hombres, organizada según principios socialmente moldados en padrones occidentales y masculinos, como los de la guerra, del individualismo, de la competencia, de la agresividad. ${ }^{51}$

Con el fin de resolver estos problemas, surgen voces que reclaman un nuevo contrato social entre género e identidad de género, cuando hablamos de homosexuales, capaces de revisar los derechos y deberes de la ciudadanía a la luz de las limitaciones comentadas. ${ }^{52}$ En ese sentido, bien nos aclara Del Río Villar, que al abordar el tema de la ciudadanía y participación social aduce que:

La democracia representativa, se complementa con un ejercicio participativo, configurando un modelo europeo de debate. También se explican cuáles son las estrategias y sinergias comunicativas junto con la formulación de iniciativas y su desarrollo: de los "planes piloto" a la viabilidad, visibilidad e implementación con continuidad, buscando la innovación y la creatividad para "enganchar" a la ciudadanía. ${ }^{53}$

Estamos de acuerdo con la postura de Villar, mismo que sus palabras hablen de un

46 VERBICARO SOARES, D. Las mujeres..., op. cit., p. 72.

47 MAQUIEIRA DÁNGELO, V. Democracia, Feminismo y Universidad en el Siglo XXI. Instituto Universitario de Estudios de la Mujer. Madrid, España, 2005, p. 284.

48 Un ejemplo para los estudios sobre la materia de género se encuentra en la creación del Centro de Estudios de las mujeres en Salamanca: continuamente se habla de sensibilización en el tema y una necesaria formación específica sobre género, algo a lo que tanto la USAL como la UPSA han decidido unirse dedicando estudios especializados. Así, la USAL cuenta con un Centro de Estudios de la Mujer, que inició realmente sus formaciones en 2002 cubriendo necesidades formativas de grado y posgrado, investigando y siendo herramienta de asesoramiento docente para la universidad en materia de género. CONTRA LOS MALOS TRATOS. Reportaje violencia de género. Tribuna Universitaria. Salamanca no 507. 24-30 Noviembre, Salamanca, España, 2008, p.12-3.

49 SEGARRA, M.; CARABÍ, Á. Nuevas Masculinidades. Construyendo nuevas masculinidades: Una introducción. Icaria, mujeres y culturas. Barcelona, Espańa: Icaria, 2000, p. 43.

50 VERBICARO SOARES, D. Las mujeres..., op. cit., p. 72.

51 DE SOUSA SANTOS, B. A crítica da razäo indolente. Contra o desperdício da experiência: para um novo senso comum. A ciência, o direito e apolitica na transição paradigmtática. Vol. 1. 2a Edição. Edições Afrontamento. Porto, Portugal, 2002, p.83.

52 VERBICARO SOARES, D. Las mujeres..., op. cit., p. 73.

53 DEL RÍO VILLAR, S. Ciudadanía activa en Europa. Proceso participativo y nuevos espacios para la comunicación. Grupo Difusión. Madrid, España, 2008, p. 29. 
contexto europeo, de un modo comparativo, Brasil podría tener en cuenta eses preceptos orientativos para desarrollar acciones basadas en esas ideas y adecuándolas a su propia realidad nacional. Donde las iniciativas novedosas, los estudios y estrategias estudiadas (previamente analizadas) para su efectiva aplicación como políticas ciudadanas, la visibilidad de los problemas enfrentados y los posibles caminos a ser seguidos para la consolidación de procesos ciudadanos participativos. Todos son ideales indispensables para la consolidación de una sociedad más justa, fraterna, solidaria y, principalmente, igualitaria.

La autora nos recuerda la importancia de la participación de la sociedad civil en el proyecto de desarrollo, estudios científicos, visualización de tema importantes socialmente en la esfera de un país. Así como en la externalización de las organizaciones no gubernamentales para generar un proceso de implementación de un sistema de participación, donde la contribución ciudadana es fundamental para concienciar una masa social de ciudadanos responsables por la manutención, el orden y la participación conjunta con el Estado para la manutención de la armonía social:

Las Organizaciones No Gubernamentales, por el hecho de ser sociedad, tienen más defensas por varias razones: conocer mejor y de modo más profunda el ámbito al que pertenecen y los intereses que defienden, llegan a los políticos con un conocimiento de la realidad de mayor calado y mantienen una relación de proximidad y cercania respecto a las personas a las que representan. Por estas razones, debemos afirmar que las ONGs si son representantes validos de la sociedad civil porque son sociedad en sí mismas. $^{54}$

Para complementar los argumentos sobre los procesos y la movilización histórica de los colectivos sociales en el ámbito de las personas LGBTs, por ejemplo en Brasil, como bien nos aclaró Bezerra en su momento: en el Gobierno del presidente Ernesto Geisel, se ha generado una represión menor a los movimientos sociales y el regreso de diversos intelectuales a nuestro país, aportando influencias que los mismos hayan tenido con novedosas tendencias de manifestaciones populares e exteriorizaciones de demandas existentes tanto en Europa como en EE.UU. ${ }^{55}$

Para Bezerra, al hacer referencia a la movilización inicial brasileña de los grupos de homosexuales, así como a las primeras organizaciones sociales, organizadas para atender y visibilizar la situación y a reivindicar los derechos de ese colectivo, nos informa que, según la realidad brasileña, ha sido en 1970. Para el autor, al citar Facchini:

Esas asociaciones pesen el hecho de reuniesen homosexuales, tenían una participación definida por los militares como "no politizada", por estar exclusivamente para la "sociabilidad". Esas primeras formas de asociación de homosexual, como por ejemplo el periódico "O Snob" de 1963-1969 y la "Associação Brasileira de Imprensa Gay" de 1967-1968, son algunos de los casos. ${ }^{56}$

En ese sentido, se ejemplifica en ese momento, a finales de la década de los 70, el Grupo Somos de Sáo Paulo se estructura en las luchas reivindicativas por los mismos derechos que a los demás ciudadanos. Con el pasar de los tiempos, otros movimientos se fortalecieron, y la organización del colectivo LGBT brasileño ha ocupado un espacio en los discursos de la sociedad. Bezerra explicita que, a partir de los años 90, organizaciones ciudadanas de participación en

54 DEL RÍO VILLAR, S. Ciudadanía activa..., op. cit., p. 35-9.

55 DOS SANTOS BEZERRA, R Discriminação por orientação sexual na perspectiva dos Direitos Humanos: um panorama da legislação, jurisprudência e açôes afirmativas no Brasil. Tesis Doctoral. Universidad de Salamanca. Salamanca, España, 2011, p. 273.

56 DOS SANTOS BEZERRA, R. Discriminação..., op. cit., p. 273. 
favor de los derechos del público LGBT se han destacado, como: la Associação Brasileira de Gays, la Associação Brasileira de Lésbicas y la Liga Brasileira Lésbicas, el Grupo Gay de Bahia, la Associação Brasileira de Gays, Lésbicas, bissexuais e transexuais. ${ }^{57}$

Del mismo modo que los temas de identidad de género y la visibilidad extendida a los travestís y a las personas transexuales tienen también, por medio de organizaciones como: Associação Nacional das Transgéneros y el Coletivo Nacional de Transexuais. ${ }^{58}$

\section{Las garantías para los Derechos Humanos y la no discriminación}

La lucha contra la discriminación por razón de orientación sexual hace parte de un importante trabajo colectivo que dependerá también de la contribución de las ONGs, como se ha visibilizados en otras sociedades internacionales. Para esa idea citamos a Verde i Aldea, que, al tratar la discriminación por razón de sexo, en el ámbito comunitario europeo, nos informa que:

La acción contra la discriminación por razón de sexo complementa los esfuerzos que, en la misma dirección, están ya llevados a término, con resultados todavía no plenamente satisfactorios, los Estados Miembros por medio de su ordenamiento interno y convenios internacionales en los que son parte. ${ }^{59}$

Fernández Sánchez comenta que la situación de Latinoamérica (cuanto a la protección de los Derechos Humanos), preceptúa un cuadro todavía preocupante. Según sus palabras:

Otras regiones del mundo, como Latinoamérica, África etc. Van estableciendo las bases de un sistema jurídico de protección internacional de los Derechos Humanos, pero sus órganos de control ejercen más funciones consultivas que decisorias y las dificultades de la puesta en práctica o la falta de adhesiones de los Estados los hacen inviables en la práctica [...] Seguimos observando, en definitiva, que los Estados, en esta materia, son terriblemente cautos. Por eso, no puede claudicarse y no pueden echarse las campanas al vuelo. Tenemos una conquista que hacer, imparable, y la Declaración Universal de los Derechos Humanos nos sirve de vigía, acechando cuanto pueda mejorar, por modesto que sea, la vida. ${ }^{60}$

Para los esfuerzos de consolidación real de los Derechos Humanos comentados, debemos reconocer que ese trabajo se hace bastante laborioso, principalmente en zonas donde la inestabilidad de Gobiernos democráticos y graves problemas en áreas como: social, económica, política, jurídica han sido implementados.

La realidad brasileña en similar situación de otros países del continente americano, así como de otras partes del mundo, sea en África o Asia. Las violaciones a los Derechos Humanos siguen siendo tema de discursos internacionales, por Organismos que objetivan, por lo menos, simbólicamente, en reivindicar logros efectivos en la protección de los dichos Derechos Universales. Para Rovira:

La lucha contra la pobreza es una cuestión material, jurídica y se combate con normas y medidas políticas y económicas nacionales e internacionales, y no sólo porque la miseria sea moralmente reprobable, sino porque es injusta [...] es necesario lograr gobiernos dinámicos, eficientes y efectivos, internacionales,

57 DOS SANTOS BEZERRA, R. Discriminação..., op. cit., p. 274.

58 DOS SANTOS BEZERRA, R. Discriminação..., op. cit., p. 274.

59 VERDE I ALDEA, J. Los Derechos Humanos y las Comunidades Europeas. $1^{a}$ Edición. Grupo Socialista del Parlamento Europeo. Madrid, España, 1989, p. 85.

60 FERNÁNDEZ SÁNCHEZ, P. A. La Desprotección internacional de los Derechos Humanos (a la luz del 50 aniversario de la Declaración Universal de los Derechos Humanos). Universidad de Huelva. Huelva, España, 1998, p. 14. 
estatales, regionales y locales que regulen y garanticen la circulación mundial fluida de las personas y capitales, evitando la exclusión y las sacudidas financieras que, en días, hunden mercados y con ellos los derechos económicos, sociales y culturales de las gentes, destrozando la sociedad civil. ${ }^{61}$

Para esa visión, podemos citar a Rovira que analiza los Derechos Humanos como derechos que:

No han caído del cielo ni se han dado todos de una vez, más bien son fruto de conquistas parciales logradas siempre cuesta arriba, frente a la pendiente. Nacen al inicio de la Edad Moderna y se convierten en uno de los indicadores principales de progreso histórico y núcleo de legitimidad de los sistemas democráticos. Derechos y deberes que, desde su formación inicial como instrumento para limitar el poder, siguen un proceso que permanece abierto de perfeccionamiento de su contenido y necesariamente también de las instituciones y técnicas que posibilitan su realización efectiva, lo que conocemos con la expresión de garantías. ${ }^{62}$

Frente a ese ideario de limitación de poderes y la necesidad de los Derechos Humanos pasaren por un proceso de ampliación de sus contenidos, es importante que tengamos en cuenta, que los mismos derechos deben adecuarse al surgimiento de las nuevas necesidades humanas, entre ellas el reconocimiento y la protección efectiva de valores relacionados, sin ninguna duda, con la enseñanza universal sobre la sexualidad humana. Del mismo modo en que se concentraron hace años esfuerzos en evidenciar los problemas de género y resguardar la sexualidad de la mujer y temas relacionados con los derechos de reproducción y de tener control y derecho sobre el propio cuerpo de la mujer.

Notaremos que, en la actualidad, es preciso enfocar trabajos conjuntos, tantos por los Estados, así como con la sociedad civil organizada, las ONGs, las empresas privadas en discutir, establecer metas, estudiar los casos enfrentados, comparar experiencias para, también, tratar de los temas de orientación sexual, tan importantes para la dignidad humana y en directa relación con los Derechos Humanos.

De acuerdo con Benedek, el respeto por los Derechos Humanos debe estar presente en los esfuerzos regionales y nacionales de cada Estado en desarrollar nos medios necesarios para el desarrollo de los mismo. El autor (como sugerencia de acciones implementadas) para tornarlos efectivos menciona que: a) la capacitación en materia de los Derechos Humanos, educación para los mismos, b) la creación de instituciones nacionales para la promoción y fiscalización, c) la creación y adecuación de programas de formación para los profesionales en distintas áreas de la sociedad para enfrentar los desafío que necesitan los Derechos Humanos para ser aplicados y en el sentido de sensibilizar para la importancia de esos mismos derechos, d) la implementación en el ámbito privado de códigos de conductas voluntarios para impedir conductas discriminatorias, e) cláusulas antidiscriminación previstas en empresas privadas que desarrollen actividades con el Poder Público de un Estado, como bien funcionan en Reino Unido y Suecia. Los esfuerzos conjuntos de los Estados y sus sociedades en buscar alternativas para proteger los Derechos Humanos. ${ }^{63}$

Para la comprensión los problemas de efectividad de los Derechos Humanos:

61 ROVIRA, A. "Gobernanza y derechos humanos". In: VV.AA.: Gobernanza Democrática. Cátedra de Estudios Iberoamericanos Jesús de Polanco. Madrid, España: Marcial Pons Ediciones, 2013, pp. 21-2.

62 Cf. ROVIRA, A. "Gobernanza y derechos humanos..., loc. cit, p. 15.

63 BENEDEK, W. Comprender os direitos humanos. Manual de educação para os direitos humanos. 1a Edição. Coimbra, Portugal: Coimbra Editora, 2014, p. 78. 
En la sociedad globalizada, la realidad se disfraza, ya no hay a un lado los defensores de la democracia y al otro los dictadores legitimados por la fuerza de armas. Se está perdiendo la claridad y los derechos (humanos, demasiados humanos) se proclaman a menudo y se defienden troceados, se infringen unos y se olvidan otros. Se discute si son sociales o individuales, si son de primera, de segunda o tercera generación, se discute mucho sobre su teoría y se trabaja poco a favor de su realización. ${ }^{64}$

Para complementar ese ideario, Bobbio previamente argumentaba que el momento más reciente de los Derechos Humanos se pasa del período de proclamarlos al período de darles efectividad.

Nuestro posicionamiento es de reconocer la viabilidad de dar una real realización y aplicabilidad de protección de los Derechos Humanos. Hemos visto, a lo largo de la historia de la humanidad, los distintos agentes que han intentado separar a las personas en diversas categorías, como si esas personas fuesen realmente distintas entre sí. Hecho ese que contradice la esencia humana, de que todos son iguales. Cuando abordamos la temática de la orientación sexual en su diversidad, nos hemos deparado con discursos donde la separación entre las personas fundamentaba y segregaba a las personas que podrían amar de un modo libre y a otro grupo al cual ese amor les era denegado.

Amar es inherente al ser humano y bajo esa esencia, no se puede obstaculizar ese amor, caso contrario, la persona humana tiene afectada sus bases esenciales.

De ese modo, no se puede admitir que los Derechos Humanos sean divididos, son derechos que se relacionan entre sí, se complementan y no existen unos sin los demás. No es admisible que se le conceda a una persona la posibilidad de ejercer la libertad de expresión, pero al mismo tiempo denegarla el derecho de expresar su ejercicio de amar a otra persona.

No se puede plegar la extensión de la libertad a una persona, de considerarla libre en una sociedad, sin al mismo tiempo no le es permitida la posibilidad de realmente ejercer esa libertad en escoger el modo en que desea vivir su sexualidad y, por supuesto, su orientación sexual homosexual. Entendemos que amar es vivir y la vida constituye el bien más importante para una persona. Por consiguiente, si una persona no vive en el amor, poco o nada le resta para sobrevivir dignamente.

En ese sentido el preámbulo de la Declaración se puede encontrar la aclaración:

La Asamblea General proclama esta Declaración universal de derechos humanos como el ideal común que todos los pueblos y todas las naciones deben alcanzar con el fin de que todas las personas y órganos de la sociedad, teniendo esta Declaración siempre presente en el espíritu, se esfuercen en promover el respeto de estos derechos y de estas libertades mediante la enseñanza y la educación, y asegurar con medidas progresistas de orden nacional e internacional su reconocimiento y aplicación universales y efectivos, tanto por parte de los Estados miembros como de los territorios de que jurídicamente dependen. ${ }^{65}$

Cuanto, a esa visión, opinaremos que el carácter universal de los Derechos Humanos y en la promoción de un ideal de respeto a los Derechos Humanos y como valor ampliamente extendido a los Estados que la firmaron en desarrollar, incluso, sus ordenamientos nacionales para adecuarlos a los preceptos establecidos por la esfera internacional de protección de los

64 Cf. ROVIRA, A. "Gobernanza y derechos humanos..., loc. cit, p. 15.

65 MESTRE CHUST, J. V. Los derechos humanos. Barcelona, Espańa: Editorial UOC, 2007, p. 11. 
mismos derechos, para que no constituyan solamente preceptos de limitada aplicabilidad.

\section{Consideraciones finales}

Creemos en la importancia de instrumentos de enseñanza y de educación para cambiar las realidades son fundamentales para enfocar esa educación para el respeto de la diversidad y la orientación sexuales homosexual.

Opinaremos de modo favorable a los argumentos presentados por López Sánchez y reconocemos los problemas enfrentados en la zona de Suramérica, en los temas de Derechos Humanos y su real efectividad. Muchos son los desafíos que todavía se presentan principalmente en Brasil, una vez que las grandes desigualdades sociales entre sus ciudadanos constituyen un serio problema para el desarrollo interno de las condiciones de bienestar social de su población.

Un número superior de ciudadanos siguen viviendo en niveles lamentables de problemas, sin acceso a una alimentación digna, a la salud pública de calidad, a un sistema de educación eficaz, a una morada. Sin comentar el poco y escaso conocimiento en pleitear, por parte de esa población, excluida de participación en sus derechos a una vida digna.

Cuanto a los temas de género y de orientación sexual, veremos que la realidad brasileña tiene problemas que dificultan en el respeto a ciertos colectivos sociales, dentro de la sociedad. Colectivos eses, por ejemplo, los homosexuales, que hace, pocos años, han notado cambios en el sentido de ver reconocidos algunos derechos fundamentales y, también, en el trato en igualdad frente a los demás ciudadanos. Lo que implica en reconocer los esfuerzos de la comunidad civil organizada en Brasil para posibilitar los cambios hacia a una realidad más inclusiva para la diversidad sexual.

\section{Referencias}

ABGLT. Manual de Comunicação LGBT. Lésbicas, Gays, Bissexuais, Travestis e Transexuais. Brasilia, Brasil, 2010.

BAILE AYENSA, J. I. Estudiando la Homosexualidad. Teoría e investigación. 1a Edición, Madrid, España: Ediciones Pirámide, 2008.

BENEDEK, W. Comprender os direitos humanos. Manual de educação para os direitos humanos. Coimbra, Portugal: Coimbra Editora, 2014.

CANCIO ÁlVAREZ, M. D. “Glosario”. In: Conde Álvarez, E. / Figueruelo Burrieza, A. / Nuño Gómez, l.: Estudios Interdisciplinares sobre Igualdad. Segunda Edición. Madrid, España: Iustel - Instituto de Derecho Público, 2011.

CEZÁRIO, J. Legislação e Jurisprudência LGBTTT. Brasília. Brasil, Instituto de Bioética, Direitos Humanos e Gênero e Associação Lésbica Feminista de Brasília Coturno de Vênus, Brasília, Brasil, 2007.

CONTRA LOS MALOS TRATOS. Reportaje violencia de género. Tribuna Universitaria. Salamanca no 507. 24-30 Noviembre, Salamanca, Espańa, 2008.

DE SOUSA SANTOS, B. A crítica da razão indolente. Contra o desperdício da experiência: para um novo senso comum. A ciência, o direito e apolítica na transição paradigmtática. Vol. 1. 2a 
Edição. Edições Afrontamento. Porto, Portugal, 2002.

DEL RÍO VILLAR, S. Ciudadanía activa en Europa. Proceso participativo y nuevos espacios para la comunicación. Grupo Difusión. Madrid, España, 2008.

DOS SANTOS BEZERRA, R. Discriminação por orientação sexual na perspectiva dos Direitos Humanos: um panorama da legislação, jurisprudência e açôes afirmativas no Brasil. Tesis Doctoral. Universidad de Salamanca. Salamanca, España, 2011.

FERNÁNDEZ SÁNCHEZ, P. A. La Desprotección internacional de los Derechos Humanos (a la luz del 50 aniversario de la Declaración Universal de los Derechos Humanos). Universidad de Huelva. Huelva, España, 1998.

FURLANI, J. "Direitos Humanos, Direitos Sexuais e pedagogia queer: o que essas abordagens têm a dizer à educação sexual?”. In: Diniz Junqueira, R. Diversidade Sexual na Educação: problematizaçôes sobre a homofobia nas escolas. Coleção Educação para todos. Brasília, Brasil, 2009.

LÓPEZ SÁNCHEZ, F. La Educación Sexual. Madrid, España: Biblioteca Nueva, 2005.

MAQUIEIRA DÁNGELO, V. Democracia, Feminismo y Universidad en el Siglo XXI. Instituto Universitario de Estudios de la Mujer. Madrid, España, 2005.

MCDOWELL, L. Género, identidad y lugar. Madrid, España: Ediciones Cátedra, 1999.

MESTRE CHUST, J. V. Los derechos humanos. Barcelona, España: Editorial UOC, 2007.

PRECIADO, B. Manifiesto Contrasexual. Colección Argumentos. Barcelona, España: Editorial Anagrama, 2011.

PROBST, E. R. A evolução da mulher no mercado de trabalho. Instituto Catarinense de PósGraduação - ICPG. Curso de Gestão Estratégica de Recursos Humanos. Florianópolis/SC, Brasil, 2003.

ROVIRA, A. “Gobernanza y derechos humanos”. In: VV.AA.: Gobernanza Democrática. Cátedra de Estudios Iberoamericanos Jesús de Polanco. Madrid, España: Marcial Pons Ediciones, 2013.

SCRIBD. Entrevista con Beatriz Preciado por Jesús Carrillo. Disponible en: <http://es.scribd. com/doc/62741654/Entrevista-com-Beatriz-Preciado-por-Jesus-Carrillo>. Acceso en: 26 de may de 2021.

SEGARRA, M.; CARABÍ, Á. Nuevas Masculinidades. Construyendo nuevas masculinidades: Una introducción. Icaria, mujeres y culturas. Barcelona, España: Icaria, 2000.

VERBICARO SOARES, D. Homossexualidade e Forças Armadas. A busca pela efetividade dos Direitos Humanos no Brasil. Trabajo de Grado. Universidad de Salamanca: Programa de Doctorado Pasado y presente de los Derechos Humanos, Salamanca, España, 2011.

VERBICARO SOARES, D. La libertad sexual en la sociedad: especial referencia a la homosexualidad en las Fuerzas Armadas Brasileñas. Salamanca, España. Tesis Doctoral. Universidad de Salamanca - USAL: Programa de Doctorado Pasado y Presente de los Derechos Humanos, 2015. 
VERBICARO SOARES, D. La viabilidad de la presentación de un servicio contratado para un establecimiento de alojamiento turístico en Salamanca para alojar estudiantes brasileños y gestión de sus asuntos académicos. C.F.G.S. Gestión de Alojamientos Turísticos. Escuela de Hostelería "Fonda Veracruz". Modulo Proyecto Final. IES Vaguada de la Palma. Salamanca, España, 2014.

VERBICARO SOARES, D. Las mujeres y las personas homosexuales en las Fuerzas Armadas. Especial referencia a las FFAA brasileñas. Trabajo fin de curso Máster Oficial en Estudios Interdisciplinares de Género. Universidad de Salamanca. Salamanca, España, 2012.

VERDE I ALDEA, J. Los Derechos Humanos y las Comunidades Europeas. 1a Edición. Grupo Socialista del Parlamento Europeo. Madrid, España, 1989.

XEGA.ORG. Juan Antonio Herrero Brasas publica su nuevo libro 'Primera Plana. La construcción de una cultura queer en España.' Disponible en: <http://xega.org/xega/noticias/xega/juan_ antonio_herrero_brasas_publica_su_nuevo_libro_primera_plana_la_construccion_de_un $>$. Acceso en: 26 de may de 2021.

YOUTUBE. Beatriz Preciado. 16 de julio de 2006. Disponible en: <http://www.youtube.com/ watch?v=W8wfc2JNMd4>. Acceso en: 26 de may de 2021.

ZANOTTI, P. Gay. La identidad homosexual de Platón a Marlene Dietrich. Colección Noema. Madrid, España: Turner Publicaciones, 2007. 